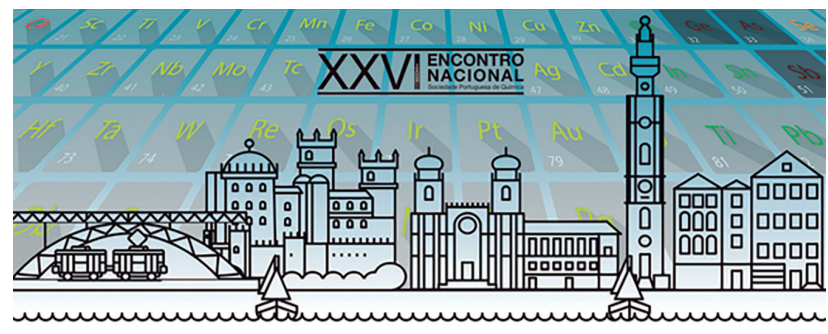

XXVI Encontro Nacional da Sociedade Portuguesa de Química

2019 é o ano do Encontro Nacional da Sociedade Portuguesa de Química. O XXVI Encontro decorrerá na Universidade do Porto, Faculdade de Ciências, de 21 a 23 de julho de 2019. Tendo em conta que o ano de 2019 é o ano internacional da Tabela Periódica, este encontro tem como mote a "Tabela Periódica” sendo de destacar os tópicos seguintes:

- A Tabela Periódica e os Elementos da Vida (saúde, alimentação e ambiente)

- Tabela Periódica Luminescente (materiais, energia e nanotecnologia)

- A Tabela Periódica na sala de aula (comunicação, cultura e ensino)

Assim, na qualidade de Presidente da SPQ e de organizador do encontro, convido todos os membros da comunidade de Química Nacional para participarem no referido Encontro e homenagear a Tabela Periódica.

Conjuntamente com as divisões e os grupos da SPQ, estamos a trabalhar num programa científico de excelência e desafiador em termos das novas áreas da química e tendências. Para isso já contamos com um leque de oradores estrangeiros de reconhecido mérito ao qual se irão juntar oradores nacionais que se têm destacado nas suas áreas de investigação. Espera-se que este encontro reúna investigadores de diferentes áreas de conhecimento da química, desde cientistas seniores a jovens cientistas, para discutir e compartilhar as suas últimas conquistas num ambiente inspirador e acolhedor. Será também uma excelente oportunidade para desfrutar da encantadora cidade do Porto, classificada como Património Mundial pela UNESCO e gozar da boa hospitalidade das gentes desta cidade. Venham todos celebrar a "Tabela Periódica” no XXVI Encontro da SPQ.

Artur Silva (Presidente da SPQ)

Mais informações disponíveis em: xxvienspq.eventos. chemistry.pt

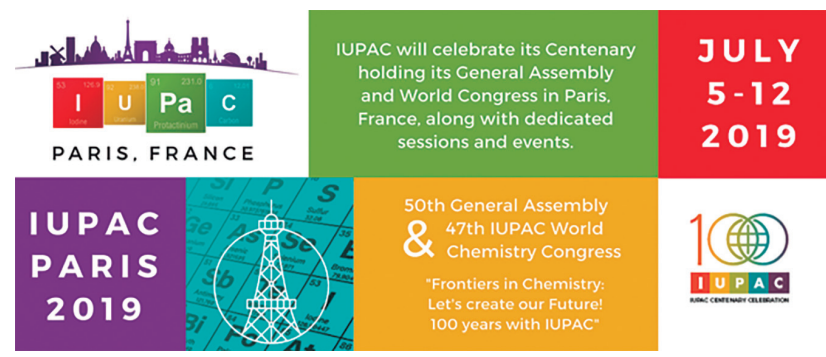

IUPAC 2019 - 47th Congress \& 50th General Assembly and Centenary Celebration

A celebração do centenário da criação da IUPAC acontecerá em Paris, de 5 a 12 de julho, em simultâneo com a 50 . Assembleia Geral e $47 .^{\circ}$ Congresso Mundial.
A IUPAC (International Union of Pure and Applied Chemistry) sucedeu à IACS (International Association of Chemical Societies) em 1919 com o objetivo de "organizar a permanente cooperação entre a as sociedades químicas dos países aderentes, coordenar os seus recursos técnicos e científicos e contribuir para o progresso da química em todos os aspetos da área”. O alcance da sua missão é evidente, e culmina com a celebração do centenário da sua existência no mesmo ano em que se celebram os 150 anos da tabela periódica.

$\mathrm{O}$ evento encontra-se dividido em duas partes: a realização da 50. ${ }^{a}$ Assembleia Geral, entre 5 e 12 de julho, e o 47. ${ }^{\circ}$ Congresso Mundial entre 7 e 12 de julho no Palais des Congrès de Paris.

- O Encontro estará dividido em diversos temas:

- Química para a vida

- Química para energia e recursos

- Química para o ambiente

- Química e sociedade

- Educação em Química

- IUPAC e a Química

- Jovens cientistas

- Simpósios especiais

Tratando-se de um encontro mundial, o evento contará com um riquíssimo painel de oradores, muitos dos quais já reconhecidos pela atribuição do prémio Nobel da Química.

O registo a custo reduzido é possível até 31 de maio de 2019.

Mais informações disponíveis em: www.iupac2019.org

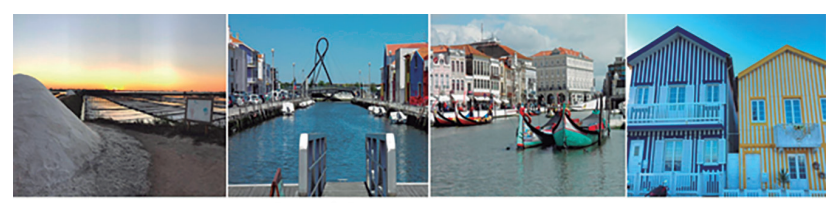

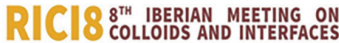

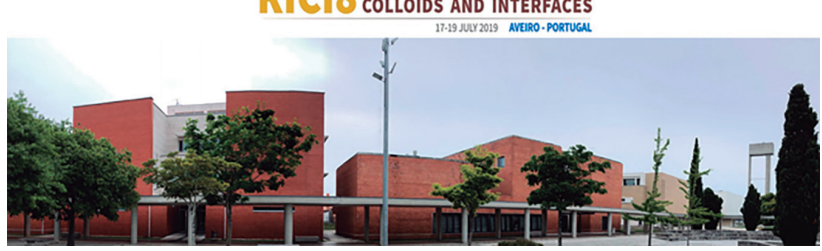

RICl8 - 8th Iberian Meeting on Colloids and Interfaces

A 8. ${ }^{a}$ Reunião Ibérica de Coloides e Interfaces (RICI) irá decorrer de 17 a 19 de julho de 2019, na Universidade de Aveiro. A RICI é uma conferência promovida pelo Grupo Especializado de Coloides e Interfases (GECI) da Real Sociedad Española de Química (RSEQ) e pela Real Sociedad Española de Física (RSEF), e o Grupo de Coloides, Polímeros e Interfaces da Sociedade Portuguesa de Química (SPQ). Esta reunião tem como objetivo reunir as comunidades espanhola e portuguesa que partilham interesses em temas relacionados com a ciência de colóides e interfaces, promovendo a discussão científica e fomentando a cooperação a nível internacional, deste modo convocando investigadores de todas as partes do mundo.

Os diversos tópicos visados pelo RICI8 incluem: 
- Coloides, biocoloides, nanopartículas e nanociência coloidal

- Superfícies, interfaces e filmes

- Polímeros, espumas, emulsões, cristais líquidos e géis

- Ciência e tecnologia de surfactantes

- Materiais avançados e sua caracterização
- Teoria e modelação aplicada a materiais moles

- Métodos experimentais em ciência coloidal e de interfaces

- Coloides e superfícies em tecnologias ambientais e de energia

Mais informações disponíveis em: www.iupac2019.org maio de 2019

5 - 10 de maio de 2019, Les Diablerets, Suíça

Tackling the Carbon Dioxide Challenge for a Sustainable Future

www.grc.org/carbon-capture-utilization-and-storage-conference/2019

16 - 17 de maio de 2019, Guimarães, Portugal

$4^{\text {th }}$ International Conference on Energy \& Environment: bringing together Engineering and Economics (ICEE 2019) coutin68.wixsite.com/icee2019

26 - 31 de maio de 2019, Cairns, Austrália

$13^{\text {th }}$ International Conference on Fundamentals of Adsorption (13FOA)

foa2019.com

29 - 31 de maio de 2019, Lisboa, Portugal

The Plastics Heritage Congress 2019

plasticsheritage2019.ciuhct.org

20 de Maio - 1 de junho de 2019, Vila Real, Portugal

1. ${ }^{\circ}$ Congresso Internacional de História da Ciência no Ensino www.utad.pt/gform/event/1o-congresso-internacional-de-historia-da-ciencia-no-ensino

\section{junho de 2019}

$2-6$ de junho de 2019, Lecce, Itália

$14^{\text {th }}$ International Symposium on Macrocyclic and Supramolecular Chemistry (ISMSC2019)

ismsc2019.eu

10 - 13 de junho de 2019, Caparica, Portugal

$3^{\text {rd }}$ International Caparica Conference in Antibiotic Resistance 2019 (IC2AR 2019)

www.ic2ar2019.com

10 - 13 de junho de 2019, Cracóvia, Polónia

EFMC-ACSMEDI MedChem Frontiers 2019

www.medchemfrontiers.org

11 - 13 de junho de 2019, Reston, Virginia, EUA

$23^{\text {rd }}$ Annual Green Chemistry \& Engineering Conference and 9th International Conference on Green and Sustainable Chemistry

www.gcande.org

16 - 20 de junho de 2019, Tessalónica, Grécia

$17^{\text {th }}$ International Conference on Chemistry and the Environment icce2019.org
17 - 18 de junho de 2019, Londres, Reino Unido

$4^{\text {th }}$ International Congress on Organic Chemistry and Advanced Drug Research

organicchemistry.pulsusconference.com

17 - 19 de junho de 2019, Porto, Portugal

EuroFoodChem XX

xxeurofoodchem.eventos.chemistry.pt

17 - 19 de junho de 2019, Valência, Espanha

$2^{\text {nd }}$ Euro Chemistry Conference

chemistry-conference.com

24 - 27 de junho de 2019, Lisboa, Portugal

$1^{\text {st }}$ International Meeting on Deep Eutectic Systems

eventos.fct.unl.pt/desmeeting2019

26 - 30 de junho de 2019, Portoroz-Portorose, Eslovénia

$6^{\text {th }}$ European Conference on Environmental Applications of Advanced Oxidation Processes (EAAOP-6) eaaop6.ki.si

julho de 2019

1 - 3 de julho de 2019, Porto, Portugal

$4^{\text {th }}$ International Conference on Natural Fibers (ICNF2019) www.icnf2019.fibrenamics.com

5 - 12 de julho de 2019, Paris, França

IUPAC 2019 - 47th Congress \& 50th General Assembly and Centenary Celebration

www.iupac2019.org

8 - 11 de julho de 2019, Caparica, Portugal

VI International Caparica Conference on Analytical Proteomics 2019 (ICAP 2019)

www.icap2019.com

8 - 12 de julho de 2019, Paris, França

International Conference on Magnetic Fluids (ICMF 2019) premc.org/conferences/icmf-magnetic-fluids

17 - 19 de julho de 2019, Aveiro, Portugal

$8^{\text {th }}$ Iberian Meeting on Colloids and Interfaces (RICI8)

rici8.eventos.chemistry.pt

22 - 26 de julho de 2019, Alicante, Espanha

$18^{\text {th }}$ International Conference on Density-Functional

Theory and its Applications

www.dft2019.es 